\title{
Overexpression of the CD155 gene in human colorectal carcinoma
}

\author{
D Masson, A Jarry, B Baury, P Blanchardie, C Laboisse, P Lustenberger, M G Denis
}

\begin{abstract}
Background and aims-The Tage4 gene (tumour associated glycoprotein E4) is overexpressed in rat colon tumours and Min mouse intestinal adenomas. The rat Tage 4 protein has approximately $40 \%$ identity with human CD155, a member of the immunoglobulin superfamily coding for a transmembrane protein capable of serving as an entry receptor for poliovirus, porcine pseudorabies virus, and bovine herpesvirus 1. Analysis of the rat Tage4 gene has revealed structural and functional similarities with the human CD155 gene. We therefore investigated expression of the CD155 gene in human colorectal carcinomas.

Methods-Overall CD155 expression was assessed by semiquantitative reverse transcription-polymerase chain reaction (RT-PCR) and immunohistochemical analysis using tissue specimens from patients with colorectal adenomas and adenocarcinomas. We also used a qualitative RT-PCR assay to determine relative expression of different splicing variants in each sample.
\end{abstract}

Results-mRNA levels of CD155 were increased in six of six colorectal cancer tissues compared with the tumour free colon mucosa. Immunohistochemical analysis revealed an increased level of CD155 protein in 12 of 12 samples. The qualitative RT-PCR assay revealed that relative expression of the different CD155 variant transcripts was similar in the different normal and cancer samples tested, indicating that this overexpression is not associated with a particular mRNA variant generated by alternative splicing of the $C D 155$ gene.

Conclusion-We have shown for the first time that the CD155 gene is overexpressed in colorectal carcinoma and that this overexpression begins at an early stage in tumorigenesis and continues to late stages.

(Gut 2001;49:236-240)

Keywords: CD155; colorectal cancer; immunoglobulin superfamily; poliovirus receptor

As our understanding of the biology of colorectal cancer progresses, new knowledge about tumorigenesis and tumour biology can be used to diagnose, treat, and prevent this type of cancer. In an initial study of an animal model for immunotherapy of colon tumours, monoclonal antibodies directed against rat colon carcinoma cells were raised. ${ }^{1}$ One of these monoclonal antibodies (E4) reacts strongly with the primary tumours and lung metastases, as determined by immunohistochemistry. The epithelia of the gastrointestinal tract and of the bronchi are only slightly stained and no staining of other normal rat tissues is observed. Initial experiments have indicated that the antigen recognised by E4 is a transmembrane glycoprotein..$^{2}$ The corresponding Tage 4 gene (tumour associated glycoprotein E4) has since been found to be overexpressed in chemically induced rat colon tumours ${ }^{4}$ and in Min mouse intestinal adenomas. ${ }^{5}$ In contrast, a barely detectable level was found in the normal adult rat and mouse tissues tested. The Tage 4 cDNA has been isolated. ${ }^{6}$ Sequence analysis using ALIGNp software revealed $40.9 \%$ identity between the Tage 4 protein and the human $\mathrm{CD} 155^{7}$ a member of the immunoglobulin (Ig) superfamily coding for a transmembrane protein capable of serving as an entry receptor for poliovirus, porcine pseudorabies virus, and bovine herpesvirus 1 . The Tage 4 gene has been mapped to rat chromosome $1 \mathrm{q} 22^{8}$ and mouse $7 \mathrm{~A} 2-\mathrm{B} 1,{ }^{9}$ regions that are homologous to the long arm of human chromosome 19 where the CD155 gene is located. ${ }^{10}$ To gain insight into the molecular mechanisms involved in its overexpression in cancer cells, we isolated and characterised the rat Tage 4 gene. ${ }^{11}$ This gene covers $\sim 15 \mathrm{~kb}$ and is composed of eight exons and seven introns. The human CD155 gene also contains seven introns in identical positions and phases. In addition, the protein encoded by the Tage 4 gene was tested for ability to mediate entry of several viruses. The results indicated that the Tage4 gene is probably orthologous to the gene for CD155. We therefore decided to analyse expression of the CD155 gene in human colorectal carcinoma (CRC). This was performed by reverse transcription-polymerase chain reaction (RTPCR) analysis and by immunohistochemistry. We also analysed relative expression of the different variants of the $C D 155$ gene by a qualitative RT-PCR assay.

\section{Materials and methods}

TISSUE SAMPLES

Tissue samples were obtained from 18 patients (10 men and eight women; mean age 66 years, range 45-90) undergoing surgical resections for primary CRC diagnosed at the Department of Surgery, Nantes University Hospital, France. Adenomas were collected from four patients: two villous adenoma (both high grade

Abbreviations used in this paper: CRC, colorectal carcinoma; RT-PCR, reverse transcription-polymerase chain reaction; CAM, cellular adhesion molecule; CEA, carcinoembryonic antigen; Ig, immunoglobulin. 
dysplasia) and two tubular adenomas (one low grade and one high grade dysplasia). Sporadic tumours were collected from 14 patients and classified according to Astler and Coller: stage B $(n=4)$, stage $C(n=7)$, and stage $D(n=3)$. The tumours were localised in the right colon $(n=5)$, transverse colon $(n=2)$, left colon $(n=3)$, sigmoid $(n=2)$, and rectum $(n=2)$. Thirteen tumours presented a moderate differentiation, and one was highly differentiated. From each patient tumour tissue and adjacent normal mucosa (about $10 \mathrm{~cm}$ from the tumour) were excised and collected. Samples were immediately processed for isolation of epithelial cells and fragments were frozen for immunohistochemistry and kept at $-70^{\circ} \mathrm{C}$ until analysis.

\section{NORMAL COLONIC EPITHELIAL CELLS AND}

CANCER CELL LINES

Normal epithelial cells were isolated from surgical specimens using a non-enzymatic dissociation technique based on the use of the calcium chelating agent EDTA, as previously described. ${ }^{12}$

HCT8R, SW620, and SW 1116 colon carcinoma cell lines were grown in RPMI-1640 containing L-glutamine and sodium bicarbonate (Sigma, Saint Quentin Fallavier, France) supplemented with $10 \%$ fetal calf serum, penicillin (100 units $/ \mathrm{ml}$ ), and streptomycin (0.1 $\mathrm{mg} / \mathrm{ml}$ ). Cells were maintained at $37^{\circ} \mathrm{C}$ in $5 \%$ carbon dioxide and passaged twice a week.

IMMUNOHISTOCHEMISTRY

Cryostat sections $(5 \mu \mathrm{m})$ were fixed in acetone and stored at $-20^{\circ} \mathrm{C}$. Before use, slides were rehydrated with phosphate buffered saline (150 $\mathrm{mM} \mathrm{NaCl}, 10 \mathrm{mM}$ phosphate buffer, $\mathrm{pH}$ 7.4). The slides were then incubated with normal goat serum for 10 minutes. Mouse monoclonal antibody D171 (Neomarkers, Union City, California, USA) directed against human $\mathrm{CD} 155^{13}$ was used as a primary antibody at a concentration of $2.5 \mu \mathrm{g} / \mathrm{ml}$ in antibody diluent containing background reducing components (Dako, Trappes, France). Alkaline phosphatase goat antimouse Ig (Sigma) was used as a secondary antibody (dilution 1:50). Following two washes with phosphate buffered saline and one with Tris buffered saline $(150 \mathrm{mM} \mathrm{NaCl}$, $10 \mathrm{mM}$ Tris $\mathrm{Cl}$, pH 7.5), staining was developed using the Vector Red alkaline phosphatase substrate (Vector, Burlingame, California, USA). After staining, tissues were counterstained with Papanicolaou. For control of specific staining an irrelevant monoclonal mouse IgG antibody was used instead of anti-CD155.

RNA PREPARATION AND REVERSE TRANSCRIPTION Total cellular RNA was extracted from frozen tissues and cultured cells with Trizol reagent (Life Technologies, Cergy Pontoise, France) according to the manufacturer's instructions.

The reverse transcription reaction and PCR amplification were performed in a Crocodile III thermal reactor (Appligene, Illkirch, France). Aerosol resistant tips were used to prevent contamination. Total RNA $(5 \mu \mathrm{g})$ was heated at $72^{\circ} \mathrm{C}$ for three minutes and then cooled on ice. It was combined with $0.5 \mu \mathrm{g}$ of random hexamers (Promega, Lyon, France), transcription buffer ( $50 \mathrm{mM}$ Tris $\mathrm{HCl}, \mathrm{pH}$ 8.3, $75 \mathrm{mM} \mathrm{KCl}, 3 \mathrm{mM} \mathrm{MgCl}, 10 \mathrm{mM}$ DTT), dNTPs (1 mM each), RNasin (50 units; Promega), and $\mathrm{RNaseH}^{-} \mathrm{M}-\mathrm{MLV}$ reverse transcriptase (200 units; Promega) in a final volume of $20 \mu \mathrm{l}$. Incubation was performed at $42^{\circ} \mathrm{C}$ for 60 minutes.

\section{SEMIQUANTITATIVE EXPRESSION OF THE CD155} GENE

Amplifications were performed with $3 \mu$ of cDNA in a total volume of $50 \mu \mathrm{l}$ containing 10 $\mathrm{mM}$ Tris $\mathrm{HCl}, \mathrm{pH} 9.0,50 \mathrm{mM} \mathrm{KCl}, 1.5 \mathrm{mM}$ $\mathrm{MgCl}_{2}, 0.1 \%$ Triton $\mathrm{X}-100,0.2 \mathrm{mg} / \mathrm{ml}$ gelatin, $200 \mu \mathrm{M}$ of each dNTP, $100 \mathrm{pmol}$ of specific primers, and 2.5 units of Taq DNA polymerase (Promega).

Primers were designed from the published sequence of the human CD155 gene. ${ }^{10}$ They were designed to amplify cDNA but not contaminating genomic DNA. The primers used to quantify expression of the CD155 gene were located in a region corresponding to the extracellular domain of the protein, which is conserved in all splice variants (fig 1). The sequences of these primers were: CD1-5' primer: 5'-TATCTGGCTCCGAGTGCTT GCC-3'; CD2-3' primer: 5'-ATCATAGCCA GAGATGGATACC-3'.

For semiquantitative amplification, each cycle was carried out at $92^{\circ} \mathrm{C}$ for 30 seconds, $62^{\circ} \mathrm{C}$ for 30 seconds, and $72^{\circ} \mathrm{C}$ for 60 seconds. The reaction was stopped after 18 cycles, in the linear range of the amplification (not shown). Following electrophoresis, amplified DNA was transferred to Hybond N+ membranes (Amersham Pharmacia Biotech, Orsay, France). The nylon filter was hybridised using alkaline phosphatase labelled probes using the AlkPhos system (Amersham Pharmacia Biotech). For CD155, a 362 bp insert amplified by PCR from HCT8R cell cDNA with oligonucleotides $\mathrm{CD} 1$ and CD2 was used as a probe.

Normalisation was performed by RT-PCR amplification and hybridisation of $\beta$-actin cDNA. Fourteen cycles $\left(92^{\circ} \mathrm{C}\right.$ for 30 seconds, $58^{\circ} \mathrm{C}$ for 30 seconds, $72^{\circ} \mathrm{C}$ for 30 seconds) were performed with oligonucleotides B1 (5'-CCTTCCTGGGCATGGAGTCCT-3') and B2 (5'-GGAGCAATGAT-CTTGATCT TC-3'). The amplified products (202 bp) were hybridised as described for CD155.

\section{QUALITATIVE ANALYSIS OF CD155 EXPRESSION}

For analysis of CD155 variants, 35 cycles of PCR were performed as described above. The primers used were located on exons 5 and 7 , respectively, on regions corresponding to each side of the transmembrane domain, where the different splice sites are located (fig 1A). The sequences of these primers were: CD5-5' primer: 5'-TCCTGTGGACAAACCAATCA ACAC-3'; CD15-3' primer: 5'-GAGGCGCT GGCATGCTCTGT-3'.

Amplified fragments were identified by size after electrophoresis on a 3\% agarose gel stained with ethidium bromide (fig 1B). For 
A

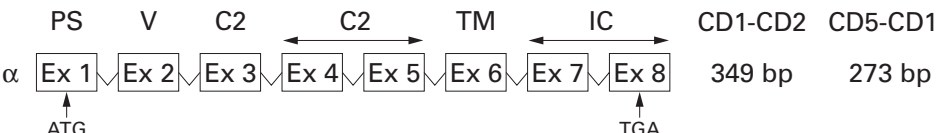

$$
\begin{aligned}
& \beta \frac{\operatorname{Ex} 1}{\operatorname{Ex} 2} \operatorname{Ex} 3 \text { Ex 4 Ex 5 } 6 \text { Ex 7 }
\end{aligned}
$$

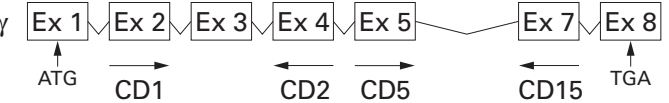

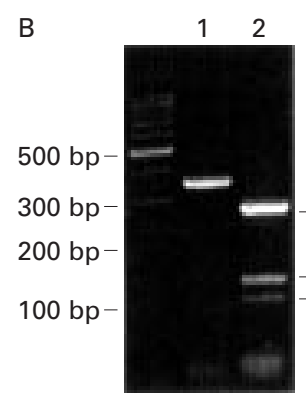

Figure 1 Overview of the different CD155 transcripts and localisation of the oligonucleotides used for reverse transcription-polymerase chain reaction (RT-PCR) analysis. (A) Exon/intron organisation of the CD155 gene. Oligonucleotides $C D 1$ and $C D 2$, used to quantify expression of the CD155 gene, are located in a region corresponding to the extracellular domain of the protein which is conserved in all splice variants. Primers CD5 and CD15, used for qualitative analysis of CD155 expression, are located on each side of the different splice sites. Part of

$\beta$ intron 6 is missing in variant $\beta$. The entire exon 6 is

$-\gamma$ missing from variant $\gamma$. The predicted size of the different amplicons is indicated. (B) Agarose gel electrophoresis of CD155 transcripts amplified by RT-PCR from SW1116 colon cancer cells. Amplification was performed with oligonucleotides $C D 1$ and $C D 2$ (lane 1) or CD5 and CD15 (lane 2). No DNA was amplified when the reverse transcription was performed in the absence of reverse transcriptase.

quantification, the bands of interest were stored as TIFF files using the BioCapt software (Vilber Lourmat, Marne la Vallée, France) and densities were determined using the EasinWin 32 software (Herolab, Fischer Scientific, Illkirch, France). The relative ratio of the different amplicons was determined for each reaction and expressed as a percentage of the overall expression. All samples were analysed at least three times.

\section{Results}

CD155 mRNA EXPRESSION IN PRIMARY CRC BY RT-PCR ANALYSIS

Firstly, we analysed expression of the human CD155 gene in human colorectal tumours by RT-PCR and DNA hybridisation. RNA was extracted from six different tumours and the corresponding adjacent normal mucosa. Semiquantitative RT-PCR analysis was performed. Expression of the CD155 gene was higher in all tumour samples compared with adjacent normal tissues, as illustrated in fig 2. Quantification of the signals obtained indicated that there was a 2-10-fold increase in amounts of CD155 mRNA in colorectal tumours compared with normal mucosa.

CD155 EXPRESSION IN PRIMARY CRC BY IMMUNOHISTOCHEMISTRY

To determine which cells overexpress the CD155 gene, immunostaining experiments were performed on frozen $5 \mu \mathrm{m}$ thick sections with monoclonal antibody D171. As illustrated in fig 3A, anti-CD155 reactivity was clearly detected in tumour samples. In each case the sections used for the negative control, which were stained with an irrelevant antibody, were negative (fig 3B). CD155 expression was localised to tumour cells and not observed in the

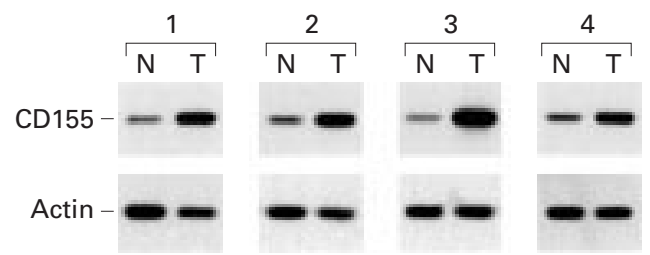

Figure 2 Increased levels of CD155 in human colorectal carcinoma. Reverse transcription-polymerase chain reaction analysis of $C D 155$ expression was performed on paired tumorous (lanes T) and non-tumorous (lanes N) colorectal tissues from four patients, as described in materials and methods.

stromal reaction under these experimental conditions. Similar staining of CD155 was identified in all adenomas as well as in all adenocarcinomas. Staining was independent of tumour grade, degree of differentiation, and localisation of the tumour. All normal colon tissues presented only weak staining of epithelial cells, and no staining was detected in the lamina propria (fig 3C).

CD155 EXPRESSION IN HUMAN CANCER CELL LINES In addition to the immunohistochemical staining experiments, D171 reactivity was observed by flow cytometry and indirect immunofluorescence with all human colon carcinoma cell lines tested so far (HT29, Colo205, SW620, HCT8R, SW1116; not shown).

CD155 EXPRESSION IN CRC BY IMMUNOBLOT ANALYSIS

To characterise further the protein detected by anti-CD155 monoclonal antibodies in human tissues, we performed western blot analyses. Proteins were extracted from four colon tumours and three colon cancer cell lines. Monoclonal antibody D171 is not suitable for western blotting. Therefore, following electrophoresis and transfer, nitrocellulose membranes were probed with monoclonal antibody 5H5 (kindly provided by Dr A Nomoto) which recognises CD155 under denaturing conditions (unpublished). This revealed an $\sim 80 \mathrm{kDa}$ protein (data not shown) which is in agreement with the reported molecular weight of the transmembrane CD155 protein. ${ }^{14}$

EXPRESSION OF CD155 SPLICING VARIANTS

The CD155 gene has been shown to be expressed as several isoforms corresponding to splicing variants. ${ }^{10}$ To analyse the relative expression of these different transcripts in CRC, a qualitative RT-PCR analysis was performed with oligonucleotides CD5 and CD15. These primers are located on exons 5 and exon 7, respectively. Agarose gel electrophoresis revealed three amplified DNA fragments (fig 1B). These different fragments were purified, cloned, and sequenced. This analysis revealed that the major band (273 bp) corresponds to the isoform containing the transmembrane domain (isoform $\alpha$ ). The lower $137 \mathrm{bp}$ and $113 \mathrm{bp}$ fragments correspond to transcripts lacking part of or the entire exon 6, respectively. They have been described as $\beta$ and $\gamma$ isoforms by Koike and colleagues. ${ }^{10}$ They lack the transmembrane domain and code for soluble forms of CD155. 

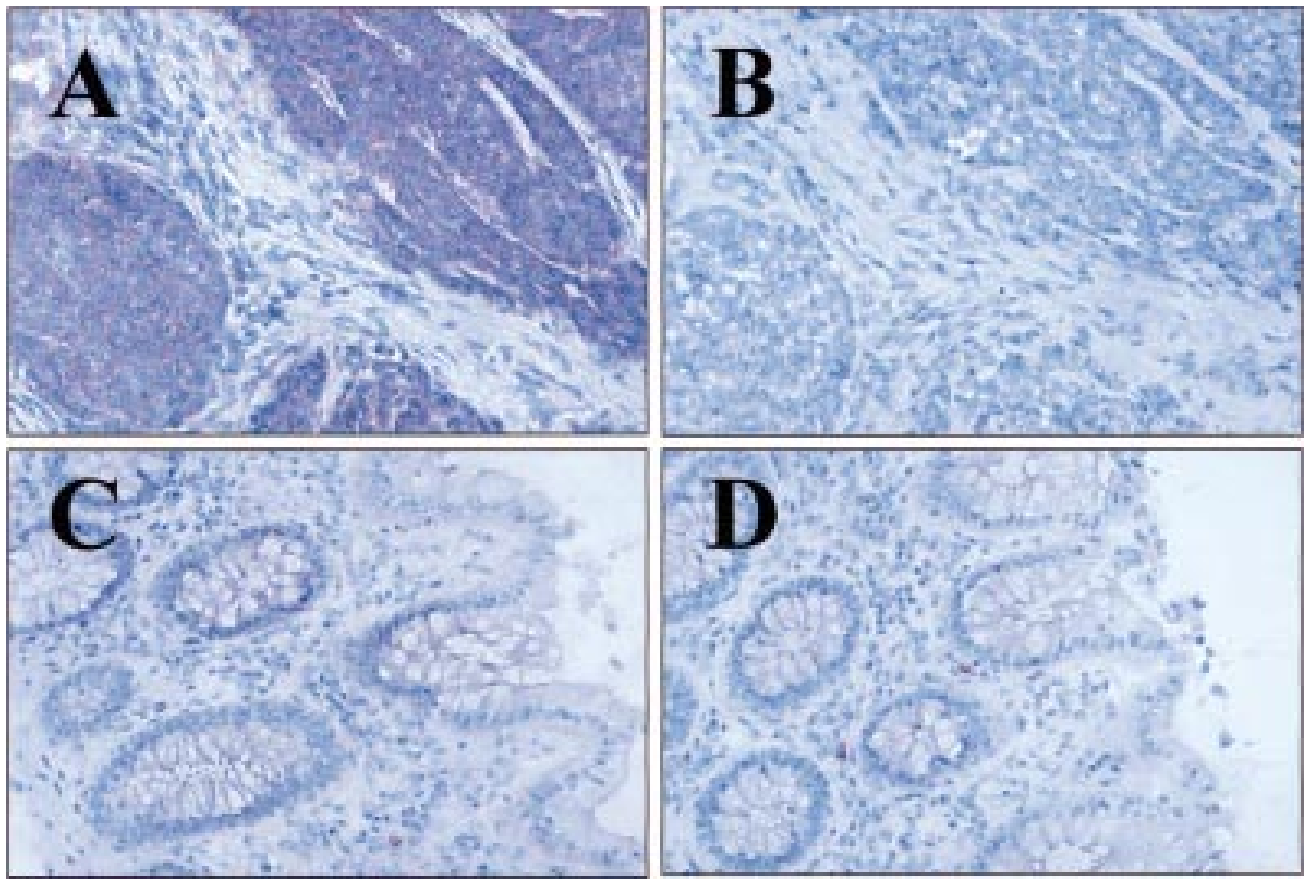

Figure 3 CD155 is overexpressed in malignant epithelial cells in colorectal carcinoma. (A) Colon adenocarcinoma shows immunoreactivity with monoclonal antibody D171 (×200). (B) Adjacent section stained with an irrelevant monoclonal antibody (×200). (C) Normal colonic tissue showing barely detectable CD155 immunoreactivity $(\times 200)$. (D) Normal section stained with an irrelevant monoclonal antibody $(\times 200)$.

cDNAs prepared from RNA extracted from tumours and normal tissues were analysed using this assay. The relative abundance of these three RNA transcripts was determined for six colon tumours and the corresponding normal mucosa. This revealed no qualitative difference between the samples studied (not shown).

All of these samples contain different cell types. Therefore, we applied this assay to preparations of normal intestinal epithelial cells isolated from surgical specimens obtained from patients undergoing colonic resection and compared the results with those obtained with human colon carcinoma cells lines. In normal epithelial cells, the transmembrane $\alpha$ isoform represents the main CD155 transcript (65$70 \%$ ) (table 1). Variants $\beta$ and $\gamma$ correspond to $25-30 \%$ and $5-8 \%$ of the CD155 mRNAs, respectively. No significant difference was observed between the three preparations tested. Similar ratios between the different splicing variants were obtained with the colon

Table 1 Relative expression (\%) of the different CD155 variants in normal epithelial cells and colon cancer cells

\begin{tabular}{llll}
\hline & \multicolumn{3}{l}{ Splicing variant } \\
\cline { 2 - 4 } & CD155a & CD155 $\beta$ & $C D 155 \gamma$ \\
\hline Normal epithelial cells & & & \\
IEC1 & $65.3(3.6)$ & $29.8(1.5)$ & $4.9(2.0)$ \\
IEC2 & $68.5(3.4)$ & $24.8(2.3)$ & $6.7(2.6)$ \\
IEC3 & $66.1(4.5)$ & $26.2(3.0)$ & $7.7(2.3)$ \\
Colon cancer cell lines & & & \\
HCT8R & $67.5(1.9)$ & $27.8(3.9)$ & $4.7(0.4)$ \\
SW620 & $63.6(2.3)$ & $31.1(0.9)$ & $5.3(0.1)$ \\
SW116 & $63.0(2.5)$ & $31.6(0.4)$ & $5.4(0.6)$
\end{tabular}

The relative ratio of the different amplicons, determined as described in material and methods, was evaluated for each reaction separately and expressed as a percentage of the overall expression in each sample. All reactions were performed at least three times. Mean (SD) values are presented. cancer cell lines analysed (table 1). These data indicate that overexpression of the $C D 155$ gene does not involve a particular isoform but most likely results from increased transcription of the gene.

\section{Discussion}

The $C D 155$ gene codes for integral membrane or soluble proteins that are members of the Ig superfamily. ${ }^{15}$ They share a V-C2-C2 domain structure. ${ }^{70}$ The members of this family have a diversity of functions but in most cases the common denominator is a recognition role at the cell surface. ${ }^{15}$ Most of these proteins are involved in cell-cell interaction during normal or pathological processes such as embryogenesis (for example, neural cellular adhesion molecule (N-CAM), and neurone-glia CAM $(\mathrm{Ng}-\mathrm{CAM})$ ), immune response (Igs, $\mathrm{T}$ cell receptors, MHC antigens), inflammation and wound healing (intercellular CAM-1 (ICAM1), CD54; vascular CAM-1 (VCAM-1), CD106), tumorigenesis and cancer metastasis (N-CAM; Ng-CAM; VCAM-1; ICAM-1; platelet endothelial CAM-1 (PECAM-1), CD31; carcinoembryonic antigen (CEA)). Some growth factor receptors also belong to the Ig family: the platelet derived growth factor receptor, colony stimulating factor 1 receptor, and fibroblast growth factor receptors. Finally, several viral infections are mediated through binding to a membrane receptor belonging to the Ig family. ${ }^{16}$

Human poliovirus infections are mostly targeted to the gastrointestinal tract through binding to the transmembrane protein coded by the $C D 155$ gene. $^{714}$ This is the only known function of the CD 155 protein at present but it probably has important biological activities. 
Indeed, CD155 belongs to a small subfamily of the Ig superfamily that includes nectin 1 , nectin 2 , and nectin 3 , all three of which have been shown to mediate cell-cell adhesion and to localise to sites of cadherin based cell junctions. ${ }^{17-19}$

As it is overexpressed in cancer cells, it is tempting to speculate that CD155 expression may play a role in neoplastic transformation and/or progression. CD155 overexpression was seen in all adenomas which are established premalignant lesions in colorectal carcinogenesis. Thus CD155 expression may play a role at early stages of tumorigenesis.

Several members of the Ig family are overexpressed in cancer cells. The $C E A$ and $N C A$ genes are overexpressed in human CRC. ${ }^{2021}$ The human $B-C A M$ gene is highly expressed in ovarian carcinomas in vivo. ${ }^{22}$ Muc 18 has been identified as a marker of tumour progression in human malignant melanoma that may have a role in the development of metastasis. ${ }^{23}$ Finally, de novo expression of ICAM-1, the major human rhinovirus receptor, ${ }^{24}{ }^{25}$ correlates with increased risk of metastasis in melanoma. ${ }^{26}$

The qualitative RT-PCR that we have developed revealed that the relative abundance of the three RNA transcripts was not significantly different between cancer cells and normal epithelial cells. This indicated that there is no difference between RNA splicing mechanisms in normal and colon cancer cells but rather that there is increased transcription of the CD155 gene in colon cancer cells.

The promoter of the human CD155 gene has been isolated. ${ }^{27}$ Solecki and colleagues ${ }^{28}$ recently reported mapping of three cis elements within the core promoter, and demonstrated that the AP-2 transcription factor is involved in transcription of the CD155 gene in $\mathrm{HeLa}$ cervical carcinoma cells. Further investigation into the regulation of the CD155 gene in colorectal cancer cells is necessary. We are also investigating the potential use of soluble CD155 molecules as tumour markers. In addition, demonstration that the CD155 gene is overexpressed at early stages of colorectal carcinogenesis should initiate studies on the function(s) of the different CD155 isoforms.

Finally, during completion of our manuscript, Gromeier and colleagues ${ }^{29}$ demonstrated that highly attenuated poliovirus recombinants can infect and propagate in cell lines derived from malignant gliomas and, most interestingly, halted tumour progression and eliminated tumours in athymic mice. They proposed that susceptibility of these malignant cells to poliovirus may be mediated by expression of the CD155 gene in glial neoplasms. Our demonstration that the CD155 gene is overexpressed in colorectal cancer cells suggests that oncolytic poliovirus recombinants may also be suitable for the treatment of colorectal cancer.

This work was supported by a grant from the Ligue Départementale (44) Contre le Cancer. B Baury is the recipient of a research fellowship from the Ligue Départementale (44) Contre le Cancer. We thank Dr Akio Nomoto (Department of Contre le Cancer. We thank Dr Akio Nomoto (Department of
Microbiology, Tokyo University, Japan) for providing the monoclonal antibodies directed against human CD155. A Jarry and B Baury contributed equally to this work.
1 Douillard JY, Laborda J, Burg C, et al. Monoclonal antibodies to a rat colon carcinoma: model for monoclonal antibody ies to a rat colon carcinoma: model for monoclonal ant

2 Chadéneau C, Denis MG, Blottière HM, et al. Characterization, isolation and amino terminal sequencing of a rat colon carcinoma-associated antigen. Int f Cancer 1991;47:903-8.

3 Chadéneau C, Lemoullac B, Cornu G, et al. Glycosylation of a novel member of the immunoglobulin gene superfamilly expressed in rat carcinoma cell lines. Int 7 Cancer 1995;61:87-91.

4 Denis MG. Characterization, cloning and expression of the Tage 4 gene, a member of the immunoglobulin superfamily. Int F Oncol 1998;12:997-1005.

5 Chadéneau C, Lecabellec M, Lemoullac B, et al. Overexpression of a novel member of the immunoglobulin superfamily in Min mouse intestinal adenomas. Int 7 Cancer 1996;68:817-21.

6 Chadéneau C, Lemoullac B, Denis MG. A novel member of the immunoglobulin gene superfamilly expressed in colon tumors. F Biol Chem 1994;269:15601-5.

7 Mendelsohn CL, Wimmer E, Racaniello VR. Cellular receptor for poliovirus: molecular cloning, nucleotide sequence, and expression of a new member of the sequence, and expression of a new member
immunoglobulin superfamily. Cell 1989;56:855-65.

8 Chadéneau C, Liehr T, Rautenstrauss B, et al. The Tage4 gene maps to rat chromosome 1q2.2. Mamm Genome $1997 ; 8: 157-8$

9 Chadéneau C, Lemoullac B, Lecabellec M, et al. Isolation and chromosomal localization of $\mathrm{mE} 4$, a novel murine gene of the immunoglobulin superfamily. Mamm Genome 1996; 7:636-7.

10 Koike S, Horie H, Ise I, et al. The poliovirus receptor protein is produced both as membrane-bound and secreted forms. EMBO ₹ 1990;9:3217-24.

11 Baury B, Geraghty RJ, Masson D, et al. Organization of the rat Tage 4 gene and herpesvirus entry activity of the encoded protein Gene 2001;265:185-94.

12 Jarry A, Vallette G, Cassagnau E, et al. Interleukin 1 and interleukin 1beta converting enzyme (caspase 1) expression in the human colonic epithelial barrier. Caspase downregulation in colon cancer. Gut 1999;45:246-51.

13 Nobis P, Zibirre R, Meyer G, et al. Production of a monoclonal antibody against an epitope on HeLa cells that is the functional poliovirus binding site. F Gen Virol 1985;66:2563-9.

14 Bernhardt G, Bibb JA, Bradley J, et al. Molecular characterization of the cellular receptor for poliovirus. Virology 1994; 199:105-13.

15 Williams AF, Barclay AN. The immunoglobulin superfamily-domains for cell surface recognition. Anпu Rev Immunol 1988;6:381-405.

16 White JM, Littman DR. Viral receptors of the immunoglobulin superfamily. Cell 1989;56:725-8.

17 Lopez M, Aoubala M, Jordier F, et al. The human poliovirus receptor related 2 protein is a new hematopoietic/ endothelial homophilic adhesion molecule. Blood 1998;92: 4602-11.

18 Takahashi K, Nakanishi H, Miyahara M, et al. Nectin/PRR: an immunoglobulin-like cell adhesion molecule recruited to cadherin-based adherens junctions through interaction to cadherin-based adherens junctions through interaction
with Afadin, a PDZ domain-containing protein. 7 Cell Biol with Afadin, a PDZ

19 Satoh-Horikawa K, Nakanishi H, Takahashi K, et al. Nectin-3, a new member of immunoglobulin-like cell adhesion molecules that shows homophilic and heterophilic cell-cell adhesion activities. F Biol Chem 2000;275: 10291-9.

20 Chi KF, Jessup JM, Frazier ML. Predominant expression of mRNA coding for nonspecific cross-reacting antigen in colorectal carcinomas. Tumour Biol 1991;12:298-308.

21 Mafune K, Saini KS, Ravikumar TS, et al. Differences in messenger RNA expression of carcinoembryonic antigen in surgical specimens of colorectal carcinoma. Tumour Biol 1992;13:330-7.

22 Campbell IG, Foulkes WD, Senger G, et al. Molecular cloning of the B-CAM cell surface glycoprotein of epithelial cancers: a novel member of the immunoglobulin superamily. Cancer Res 1994;5414:5761-5.

23 Lehmann JM, Riethmuller G, Johnson JP. MUC18, a marker of tumor progression in human melanoma, shows sequence similarity to the neural cell adhesion molecules of the immunoglobulin superfamily. Proc Natl Acad Sci USA 1989;86:9891-5.

24 Greve JM, Davis G, Meyer AM, et al. The major human rhinovirus receptor is ICAM-1. Cell 1989;56:839-47.

25 Staunton DE, Merluzzi VJ, Rothlein R, et al. A cell adhesion molecule, ICAM-1, is the major surface receptor for rhinoviruses. Cell 1989;56:849-53.

26 Johnson JP, Stade BG, Holzmann B, et al. De novo expression of intercellular-adhesion molecule 1 in melanoma correlates with increased risk of metastasis. Proc Natl Acad Sci USA 1989;86:641-4

27 Solecki D, Schwarz S, Wimmer E, et al. The promoters for human and monkey poliovirus receptors. Requirements for basic and cell type-specific activity. F Biol Chem 1997;272: 5579-86.

28 Solecki D, Wimmer E, Lipp M, et al. Identification and characterization of the cis-acting elements of the human CD155 gene core promoter. F Biol Chem 1999;274:1791-800.

29 Gromeier M, Lachmann S, Rosenfeld MR, et al. Intergeneric poliovirus recombinants for the treatment of malignant poliovirus recombinants for the treatment of mad Sci USA 2000;97:6803-8.
glioma. Proc Natl Acad 